\title{
THE GENERALIZED MAYER THEOREM IN THE APPROXIMATING HAMILTONIAN METHOD
}

\author{
A. P. BAKULEV, ${ }^{1}$ N. N. BOGOLUBOV $(\mathrm{Jr} .)^{2}$ AND A. M. KURBATOV ${ }^{2}$
}

(Received 26 September 1984)

\begin{abstract}
With the help of the generalized Mayer theorem we obtain an improved inequality for free energies of model and approximating systems, where only "connected parts" over the approximating Hamiltonian are taken into account. For a concrete system we discuss the problems of convergence of appropriate series of "connected parts".
\end{abstract}

\section{Introduction}

In recent years, the approximating Hamiltonian method has become widely used as a method for investigating model systems of statistical mechanics. The main advantage of this method is the possibility of calculating the free energy of a model exactly, in the sense of the thermodynamics limit $N \rightarrow \infty, V \rightarrow \infty$, $v \equiv V / N=$ const. The principal ideal of the method is connected with the name of N. M. Bogolubov, who has suggested replacing the operators of dynamical variables, which asymptotically (when $V \rightarrow \infty$ ) commute with the whole algebra of local observables, by $C$-numbers ( $c f$. Bogolubov $[1,2]$ ). It should also be mentioned that N. M. Bogolubov has given the first rigorous mathematical proof of the asymptotic accuracy of the method for the BCS-model of superconductors at zero temperature (Bogolubov [3]). A comprehensive mathematical formulation of the approximating Hamiltonian method has been suggested in a series of works by Bogolubov (Jr.) [4, 5], who has managed to single out a special class of model Hamiltonians (with separable limited interaction) admitting asymptotically exact

\footnotetext{
${ }^{1}$ Physical Department, Moscow State University, Moscow, USSR.

${ }^{2}$ V. A. Steklov Institute of Mathematics, 117333 Moscow, USSR.

(c) Copyright Australian Mathematical Society 1985, Serial-fee code 0334-2700/85
} 
solutions at arbitrary temperature $(\theta)$. In 1978, Kurbatov and Sankovich proposed an improvement of this theory. These authors have estimated the difference between the free energies $\left(f_{V}\right)$ of the model Hamiltonian $(\hat{H})$ and the approximating Hamiltonian $(\hat{H}(C))$ with the use of Bogolubov and Golden-Thompson inequalities (only the first has been frequently used) and made the following evaluation (see Kurbatov and Sankovich [6]):

$$
-\frac{\theta}{V} \ln \left[\left\langle\exp \frac{\hat{H}(C)-\hat{H}}{\theta}\right\rangle_{H(C)}\right] \leqslant f_{V}\{H\}-f_{V}\{H(C)\} \leqslant\left\langle\frac{\hat{H}-\hat{H}(C)}{V}\right\rangle_{H(C)} .
$$

Here $\langle\cdots\rangle_{\Gamma}$ means average over the grand canonical ensemble:

$$
\langle A\rangle_{\Gamma}=\operatorname{Tr}[\hat{A} \exp (-\hat{\Gamma} / \theta)] / \operatorname{Tr}[\exp (-\hat{\Gamma} / \theta)] .
$$

As a rule, the approximating Hamiltonian $\hat{H}(C)$ is a quadratic form with respect to creation and annihilation operators $\left(\hat{a}^{+}\right.$and $\hat{a}$, see Bogolubov [3] and Bogolubov (Jr.) [4]). For averages of products of $\hat{a}^{+}$and $\hat{a}$ over such a Hamiltonian, the Wick-Bloch-De-Dominicis theorem is valid. This fact provides us with the main method of calculating averages appearing in (1). But a direct calculation of the left part in (1) is rather hard. The generalized Mayer theorem allows us to rewrite the left part of (1) in a more convenient form.

\section{The generalized Mayer theorem}

Take $M$ numbered points and arbitrarily divide these points into groups $\left\{C_{l}\right\}$ with every point belonging to one and only one group. Next consider the operation $W$, which assigns a number $W\left(G_{M}\right)$ to each such division $G_{M}$, and which has the following properties:

(1) $W\left(G_{M}\right)$ does not depend on the numbering of points in $G_{M}$;

(2) $W\left(G_{M}\right)=\prod_{C_{l}} f_{l}$-multiplication is over all groups $C_{l}$ from $G_{M}, f_{l}$ being the number corresponding to $C_{l}$.

Now consider $F_{M}=\Sigma_{G_{M}} W\left(G_{M}\right)$, where summation covers all possible divisions $G_{M}$, and define the following functions:

$$
F(x)=1+\sum_{M=1}^{\infty} F_{M} \frac{x^{M}}{M !} ; \quad f(x)=\sum_{l=1}^{\infty} f_{l} \frac{x^{\prime}}{l !} .
$$

Then the theorem states that $F(x)=\exp (f(x))$. This theorem generalizes the well-known Mayer's theorem (the first, see J. Mayer and M. G. Mayer [7]), since for its formulation it is not necessary to define the term "diagram". This allows us to depart from the operator structure of a concrete Hamiltonian and consider the 
problem in general. The generalized theorem has a pure combinatoric character. Its proof is almost the same as Mayer's and that is why we omit it.

\section{Application of the theorem in the approximating Hamiltonian method}

In order to transform the left part of (1) with the help of the generalized Mayer theorem let us consider the average $\left\langle\hat{D}^{M}\right\rangle_{H(C)}$ where $\hat{D}=\hat{D}(C)=\hat{H}-\hat{H}(C)$. In accordance with the Wick-Bloch-De-Dominicis theorem, this average breaks down into a sum of products of pair averages $\left\langle\hat{a}^{+} \hat{a}\right\rangle_{H(C)},\left\langle\hat{a}^{+} \hat{a}^{+}\right\rangle_{H(C)},\langle\hat{a} \hat{a}\rangle_{H(C)}$. In any summand we can distinguish operators $\hat{a}\left(\hat{a}^{+}\right)$, corresponding to different operators $\hat{D}_{1}$ from $\hat{D}^{M}=\hat{D}_{1} \cdots \hat{D}_{M}$. If the operators $\hat{a}, \hat{a}^{+}$from the average $\left\langle\hat{a}^{+} \hat{a}\right\rangle_{H(C)}$ (or $\langle\hat{a} \hat{a}\rangle_{H(C)}$, or $\left\langle\hat{a}^{+} \hat{a}^{+}\right\rangle_{H\left(C_{2}\right)}$ ) belong to different operators $\hat{D}_{l}, \hat{D}_{j}$, then we shall say that these operators $D_{1}$ and $\hat{D}_{\hat{l}}$ are connected by the average $\left\langle\hat{a}^{+} \hat{a}\right\rangle_{H(C)}$. We shall also say that the operation $\hat{R}$, is connected with operator $\hat{D}_{\text {, }}$ if there is a chain $\hat{D}_{i} \leftrightarrow \hat{D}_{k} \leftrightarrow \cdots \leftrightarrow \hat{D}_{l} \leftrightarrow \hat{D}_{j}$ in which every pair $\hat{D}_{k} \leftrightarrow \hat{D}_{l}$ is connected by a pair average $\left\langle\hat{a}^{+} \hat{a}\right\rangle_{H(C)}$, or $\langle\hat{a} \hat{a}\rangle_{H(C)}$, or $\left\langle\hat{a}^{+} \hat{a}^{+}\right\rangle_{H(C)}$. Then we can extract from all the summands of $\left\langle\hat{D}^{M}\right\rangle_{H(C)}$ those summands in which every $\hat{D}_{i}$ is connected with all the others; the sum of all such summands from $\left\langle\hat{D}^{M}\right\rangle_{H(C)}$ we shall call the "connected part" $\left\langle\hat{D}_{M}\right\rangle$ of $\left\langle\hat{D}^{M}\right\rangle_{H(C)}$. Now it must be clear that if for the operation $W$ from Mayer's theorem we make the assignment $f_{l}=\left\langle D^{\prime}\right\rangle^{c}$ then $F_{M}=\left\langle\hat{D}^{M}\right\rangle_{H(C)}$ and in accordance with the theorem we have

$$
\langle\exp (-\hat{D} / \theta)\rangle_{H(C)}=\exp \left(\sum_{l=1}^{\infty}\left\langle D^{\prime}\right\rangle^{c}(-\theta)^{-1} /(l) !\right) .
$$

Taking into account that $\left\langle D^{1}\right\rangle^{c}=\langle\hat{D}\rangle_{H(C)}$ we obtain the improved form of (1):

$$
\left(\frac{1}{V}\right) \sum_{l=1}^{\infty} \frac{\left\langle D^{l}\right\rangle^{c}}{l !}(-\theta)^{1-l} \leqslant f_{V}\{H\}-f_{V}\{H(C)\}-\left\langle\frac{\hat{H}-\hat{H}(C)}{V}\right\rangle_{H(C)}
$$

Then we can conclude that for asymptotic accuracy of approximation it is sufficient to demand that the series in (4) should be convergent and its sum should vanish when $V \rightarrow \infty$. Such a situation can evidently occur only for some special values of parameters, which we shall denote by $\bar{C}$. As was pointed out by Bogolubov (Jr.) [4], and follows directly from Kurbatov and Sankovich [6], the best approximation parameters minimize the function:

$$
f_{\text {appr }}(C)=f_{V}\{H(C)\}+\left\langle\frac{H-H(C)}{V}\right\rangle_{H(C)} .
$$


As an example of the application of the improved theory, let us consider the following Hamiltonian:

$$
\hat{H}=\hat{T}+\frac{1}{V} \sum_{p_{1} p_{2}} K\left(p_{1} p_{2}\right) \hat{J}^{+}\left(p_{1}\right) \hat{J}\left(p_{2}\right) .
$$

Here $\hat{T}$ is an ordinary "kinetic" term $\left(\hat{T}=\Sigma_{p} \in(p) \hat{a}_{p}^{+}, \hat{a}_{p}\right)$ and operators $\hat{J}(p)$ âre bouinued quadraiic forms with respect to $\hat{a}_{p}^{+}$and $\hat{a}_{p}\left(\|\hat{J}(p)\| \leqslant M_{1}\right)$. The interaction potential $K$ is assumed to satisfy

$$
\frac{1}{V^{2}} \sum_{p_{1} p_{2}}\left|K\left(p_{1} p_{2}\right)\right| \leqslant M_{2} \text {. }
$$

For the approximating Hamiltonian $\hat{H}(C)=\hat{H}-\hat{D}(C)$ with

$$
\hat{D}(C)=\frac{1}{V} \sum_{p_{1} p_{2}} K\left(p_{1} p_{2}\right)\left[\hat{J}\left(p_{1}\right)-C\left(p_{1}\right)\right]^{+}\left[\hat{J}\left(p_{2}\right)-C\left(p_{2}\right)\right],
$$

it can be shown that when $|C(p)| \leqslant M_{3}$ then $\|\hat{D}\| \leqslant M_{2}\left(M_{1}+M_{3}\right) V$. Therefore the series

$$
\left\langle\exp \frac{\alpha \hat{D}(C)}{\theta}\right\rangle_{H(C)}=\sum_{M=0}^{\infty} \frac{\left\langle\hat{D}^{M}\right\rangle_{H(C)}}{\theta^{M} M !} \alpha^{M},
$$

converges absolutely at any finite $\alpha$ to an analytic function, which we shall denote by $F(\alpha)$. Besides that,

$$
F(\alpha) \geqslant \exp (-|\alpha|\|\hat{D}\| / \theta)>0
$$

and so $\psi(\alpha)=-(\theta / V) \ln (F(\alpha))$ is also an analytic function. This means that the series

$$
\sum_{l=1}^{\infty} \frac{\left\langle D^{\prime}\right\rangle^{c}}{l !} \frac{\alpha^{l}}{\theta^{\prime}}=\psi(\alpha)
$$

converges absolutely at any finite $\alpha$, in particular at $\alpha=-1$. That is why the corresponding series in (4) in our case also converges absolutely and its sum varies from zero to $-2 M_{2}\left(M_{1}+M_{3}\right)^{2}$.

We would like to obtain the following result: for some special parameters $C(p)=\bar{C}(p)$ the sum of this series vanishes when $V \rightarrow \infty$. Let us find these parameters from the minimum condition for $f_{\text {appr }}(C): \partial f_{\text {appr }}(C) / \partial C(p) \mid \bar{C}_{(p)}=0$. Then we have

$$
\bar{C}(p)=\langle\hat{J}(p)\rangle_{H(C)} .
$$

For such values of approximating parameters, one can easily estimate the magnitude of $\left\langle D^{\prime}\right\rangle^{c}$. Indeed, in this case one must take into account averages only between those operators $\hat{a}_{p}\left(\hat{a}_{p}^{+}\right)$which belong to different operators $\hat{D}$, since 
averages between operators from one $\hat{D}$ produce vanishingly small contributions (for example,

$$
\begin{aligned}
& \frac{1}{V}\langle\hat{D}\rangle_{H(C)} \\
& \quad=\frac{1}{V^{2}} \sum_{p} K(p, p) \times\left(\left\langle\hat{J}^{+}(p) \times \hat{J}(p)\right\rangle_{H(C)}-\langle\hat{J}(p)\rangle_{H(C)}\left\langle\hat{J}^{+}(p)\right\rangle_{H(C)}\right) \\
& \quad=O(1 / V) \rightarrow 0 \text { when } V \rightarrow \infty) .
\end{aligned}
$$

As a consequence, the number of summations over the quasi-continuous spectrum $(p)$ in $\left\langle D^{l}\right\rangle^{c}$ appears not to be greater than 1 . Every summation over $p$ produces a multiplier $V /(2 \pi h)^{3}$ and every $\hat{D}$ a multiplier $1 / V$. Therefore we can conclude that the magnitude of $\left\langle D^{\prime}\right\rangle^{c}$ is finite when $V \rightarrow \infty$. So we obtain: $(1 / V)\left\langle D^{\prime}\right\rangle^{c} \rightarrow 0$ when $V \rightarrow \infty$. That is why we conclude that, for parameters $\vec{C}(p)$ which realize the absolute minimum of $f_{\text {appr }}(C)$ (and therefore satisfy equation (10)), the following equality is valid:

$$
\lim _{V \rightarrow \infty} f_{V}\{H\}=\lim _{V \rightarrow \infty} f_{\text {appr }}(\bar{C})=\lim _{V \rightarrow \infty} f_{V}\{H(\bar{C})\} .
$$

The role of the selfconsistent equations (10) appears to be the following: members with $C(p)$ compensate for contributions of averages between operators $\hat{a}\left(\hat{a}^{+}\right)$ from one operator $\hat{D}$. It should be mentioned that in earlier works on the method, equations of selfconsistency have been derived from the condition of compensating some type of diagrams which have been called "dangerous". Now we obtain a similar result, but our method is rigorous.

\section{Acknowledgements}

N. N. B. (Jr.) and A. M. K. would like to thank Professor Abdus Salam, the International Atomic Energy Agency and UNESCO for hospitality at the International Centre for Theoretical Physics, Trieste, where this investigation was finished. We would like to thank Professor C. A. Hurst for hospitality and useful discussions and also Mr. I. Bray for help in the preparation of this article for publication.

\section{References}

[1] N. N. Bogolubov, "Energy levels of a non-ideal Bose-Einstein gas", Vestntk Moskov. Univ. No. 7 (1947), 43-56.

[2] N. N. Bogolubov, "A new method in the theory of superconductivity. I", Zh. Eksper. Teoret. Fiz. 34 (1958), 58-65; English transl., Soviet Phys. JETP 7 (1958), 41-50. 
[3] N. N. Bogolubov, Joint Institute for Nuclear Research, Dubna, preprint 511 and 99 (1960).

[4] N. N. Bogolubov Jr., "On model dynamical systems in statistical mechanics", Phys. 32 (1966), 933-944.

[5] N. N. Bogolubov Jr., A method for studying model Hamiltonians (Pergamon Press, Oxford-New York, 1972), 92-107.

[6] A. M. Kurbatov and D. P. Sankovich, "Consistency equations in the approximating Hamiltonian method", Joint Institute for Nuclear Research, Dubna, communication 17-10324 (1980); Teoret. Mat. Fiz. 42 (1980), 392-405; English transl. Theoret. and Math. Phys. 42 (1980), 258-266.

[7] J. Mayer and M. G. Mayer, Statistical mechanics (Wiley, New York, 1940), 274-279. 